\title{
AN EXTENDED MODEL FOR ELECTRON SPIN POLARIZATION IN PHOTOSYNTHETIC BACTERIA
}

\author{
Andrea L. Morris" ${ }^{*}$, James R. Norris"+, and Marion C. Thurnauer \\ "Chemistry Division, Argonne National Laboratory, Argonne, IL 60439
}

and

${ }^{+}$Departrnent of Chemistry, University of Chicago, Chicago, IL 60637

\section{DISCLAIMER}

This report was prepared as an account of work sponsored by at agency of the United States Government. Neither the United States Government nor any agercy thereof, nor any of their employees, makes any warranty, express or implied, or assumes any legal liability or responsibility for the accuracy, completeness, or usefulness of any information, apparatus, product, or process disclosed, or represents that its use would not infringe privately owried rights. Reference herein to any specific commercial product. process, or service by trade name, trademark. manufacturer, or otherwise does not necessarily constitute or imply its endorsement, recommendation, or favoring by the United States Government or any agen : thereof. The views and opinions of authors expressed herein do not necessarily state or reflect those of the United States Government or any agency thereof.

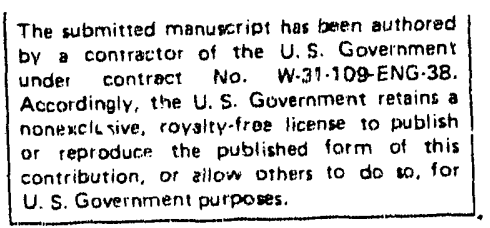




\section{Introduction}

The primary process of photosynthesis converts solar energy to chemical energy through light induced charge separation. In bacterial photosynthesis ${ }^{1}$, the best characterized photosynthetic process, pigments in the antenna proteins are excited by the absorption of visible light. This excited state is transferred to a bacteriochlorophyll dimer-.- $\mathrm{P}_{870}$, which is located in a nearby reaction center. Following this, the primary charge separation occurs; $P_{\mathbf{8 7 0}}$, subsequently referred to as $P$, is oxidized and the intermediate acceptor, I, a bacteriopheophytin, is reduced. (See figure 1.) This occurs within three picoseconds. After the bacteriopheophytin has been reduced, the reaction center is in a radical pair state, $\mathrm{P}^{+} \mathrm{B} \mathrm{I} \mathrm{FeQ}\left(\mathrm{P}^{+} \mathrm{I}^{-}\right)$. Where $\mathrm{B}$, known as the accessory bacteriochlorophyll, is situated betw een $P$ and $I$. Because the charge transfer is so rapid, the initial radical pair is born as a coherent, nonstationary singlet state. This singlet radical pair state, given sufficient time, acquires triplet character. The charge is then transferred to the iron-ubiquinone complex, FeQ. During natural photosynthesis, the charge separated state $P^{+} B \mid F e Q^{-}\left(P^{+} Q^{-}\right)$occurs approximately 200 picoseconds after the excitation of $P$. The iron can be decoupled from the quinone during preparation of the reaction center protein. In this case the electron transfer from the intermediary acceptor, $I$, to the quinone is found to take about 4 nanoseconds at room temperature. The back reactions from $\mathrm{P}^{+} I^{-} \mathrm{FeQ}$ are all relatively slow. The forward electron transfer rates are somewhat faster at lower temperatures. ${ }^{2}$

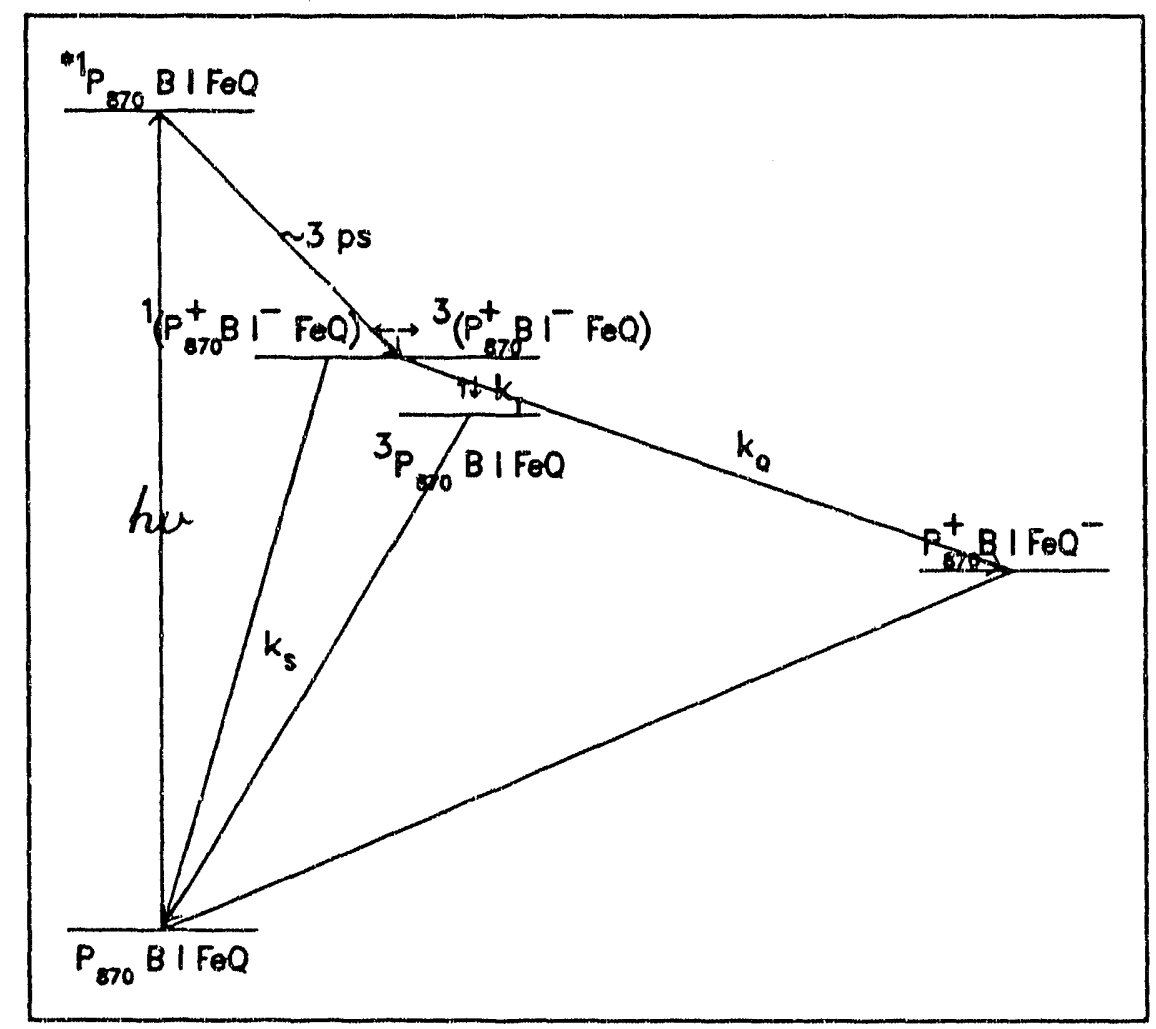

Figure 1

Electron Transfer Sequence in Bacterial Reaction

Centers 
We have utilized the energetics of the first $\left(\mathrm{P}^{+} \mathrm{I}^{-}\right)$radical pair's states to simulate electron spin polarized EPR spectra of the second radical pair in iron decoupled reaction centers of Rhodobacter sphaeroides. Previous treatments ${ }^{3,4,5,6}$ of the electron spin polarization (ESP) have made one of two assumptions (CIDEP only or CRPP only) leading to an uncoupling of the density matrix equations for the two radical pairs. In the CIDEP (chemically induced dynamic electron polarization) only calculation ${ }^{3,4,5}$, it is assumed that there are no magnetic interactions on the second radical pair, $\mathrm{P}^{+} \mathrm{Q}^{-}$. The populations of the second radical pair's eigenstates are unchanging in time after the electron transfer to the quinone is complete, and their distribution is dependent only on the magnetic interactions of the first radical pair $\left(\mathrm{P}^{+} \mathrm{I}^{\prime}\right)$. In the CRPP (correlated radical pair polarization) only calculation ${ }^{6}$, it is assumed that the initial radical pair is too short lived for the populations of its eigenstates to be significantly affected by singlet-triplet mixing due to its dipolar and exchange interactions. When the electron is transferred (to $Q$ ) the populations remain essentially as they were created, specifically the second radical pair state is initially pure singlet. In this case, the important interactions, those which cause singlet and triplet mixing, are on the second radical pair.

Simulations with interactions on only one pair, either the CRPP or the CIDEP mechanism separately, have not been completely satisfactory. A good simulation of an X-band protonated radical pair spectrum (iron decoupled) was obtained using the CIDEP mechanism, but some of the g-values and linewidths required for the best fit deviated from previously determined experimental values. Also, the relative magnitudes of $D$ (dipolar interaction) and $J$ (exchange interaction) required for a good fit were much larger than generally expected. ${ }^{5}$ The CRPP mechanism was used to simulate the same spectrum. ${ }^{6}$ Most of the parameters (D, J, dipolar angles, Q- linewidth) were in general agreement with acceptable or experimentally determined values. However, the $\mathrm{P}^{+}$linewidth used in the simulation was narrower than observed experimentally for oxidized $\mathrm{P}^{+} \mid \mathrm{FeQ} .^{7}$ In addition, one cannot necessarily assume that the $\mathrm{P}^{+} \mathrm{I}^{-}$ radical pair found in iron decoupled reaction centers is too short-lived to give rise to polarization through CIDEP. ${ }^{6}$ The lifetime of $\mathrm{P}^{+} \mathrm{I}^{-}$obtained for iron decoupled reaction centers was longer than that in native reaction centers. ${ }^{8}$ Also, Gunner et al. ${ }^{9}$ observed electron spin polarization on $\mathrm{P}^{+}$in reaction centers in which the native ubiquinone acceptor was replaced with various quinones. One effect of the quinone replacement was a change in the $\mathrm{P}^{+} \mathrm{I}^{-}$ lifetime. When the quinone was replaced, electron spin polarization on $\mathrm{P}^{+}$was only observed if the lifetime of $\mathrm{P}^{+} \mathrm{I}$ was appreciably lengthened relative to that in native reaction centers. Recently, electron spin polarized EPR spectra of $\mathrm{P}^{+} I \mathrm{Q}^{-}$have been obtained from protonated, deuterated and partially deuterated bacterial reaction center samples at both $\mathrm{X}$ - and Q-band microwave frequencies. ${ }^{10}$ These spectra have greatly enhanced the amount of experimental 
dita available to test the simulations, and preliminary attempts to simulate all these spectra with the same set of acceptable parameters indicated that neither the CRPP or CIDEP treatments alone would suffice.

We developed a genaral model ${ }^{11}$ which includes contributions from both CIDEP and CRPP. In this paper, we apply this model to sequential electron transfer in photosynthetic bacteria. Our model calculates the density matrix for the $\mathrm{P}^{+} \mathrm{I}^{-}$radical pair and transfers the polarization as it develops to the $\mathrm{P}^{+} \mathrm{Q}^{-}$radical pair. We illustrate several possible cases. One case is equivalent to CIDEP; no interactions are included on the secondary radical pair, $\mathrm{P}^{+} \mathrm{C}^{-}$. Another approximates CRPP by either increasing the transfer rate from $\mathrm{P}^{+} \mathrm{I}^{-}$to $\mathrm{P}^{+} \mathrm{Q}^{-}$or restricting interactions to the secondary radical pair, $\mathrm{P}^{+} \mathrm{Q}^{-}$. Others allow interactions on both the primary and secondary radical pairs with various transfer rates.

\section{Theory and Discussion}

Although they have been presented before, we review for comparison and a new perspective the methods used to simulate the polarized spectrum (X-band, protonated) of the $\mathrm{P}^{+} \mathrm{Q}^{-}$radical pair of Rhodobacter sphaeroides. The evolution of the density matrix for both the CRPP and the CIDEP treatments is described by

$$
\frac{d}{d t} p_{1}(t)=-\frac{i}{\hbar}\left[H_{1}, \rho,(t)\right]
$$

Where $P_{i}(t)$ is the density matrix and $\boldsymbol{H}_{\mathrm{i}}(\mathrm{t})$ is the Hamiltonian for radical pair $\mathrm{i}$. It is important to note that although the treatment of CIDEP only cases and the treatment of CRPP only cases are considered to be different treatments, the CRPP method can be used to treat noninteracting radical pairs (generally the domain of CIDEP) quite well. EPR spectra are simulated by calculating the magnetic field at which the energy gap for an allowed transition will occur. The intensity of a transition at this field is proportional to the difference in the populations of the states of the transition multiplied by the transition probability. For non-interacting radical pairs the high field eigenstates are $\Psi_{1}=B\left(P^{+}\right) \alpha\left(Q^{-}\right), \quad \Psi_{2}=\alpha\left(P^{+}\right) B\left(Q^{-}\right), T_{+}=\alpha\left(P^{+}\right) \alpha\left(Q^{-}\right)$, $T_{-}=B\left(P^{+}\right) B\left(Q^{-}\right)$. Notice that $\rho_{\alpha \alpha}\left(Q^{-}\right)=\rho_{Y_{1} \Psi_{1}}$ and $\rho_{B B}\left(Q^{-}\right)=\rho_{Y_{2} Y_{2}}$, but $\rho_{\alpha u}\left(P^{+}\right)=\rho_{\mathbf{Y}_{2} \Psi_{2}}$ and $\rho_{B B}\left(P^{+}\right)=\rho_{1}{ }_{1}$. The $\alpha$ and $B$ subscripts on $\rho$ refer to only one electron which is specified by $\mathrm{P}^{+}$or $\mathrm{Q}^{\prime}$, while the $\Psi_{1}$ and $\Psi_{2}$ subscripts refer to both the $\mathrm{P}^{+}$and the $\mathrm{Q}^{-}$electrons. $A$ transition from an $\alpha$ to a $B$ state on $P^{+}$, the transition between $\Psi_{p^{+} Q^{-}, 1}$ and $T_{-}$in fig. 2(a), occurs at the same energy as a transition from a $B$ to an $\alpha$ state on $\mathrm{P}^{+}$, the transition between $\mathbf{T}_{\mathrm{P}^{+} \mathrm{Q}^{-}, 2}$ and $T_{+}$in fig 2(a), and they have equal transition probabilities. Therefore, the shape of the spectrum depends only on the population differences $\rho_{B B}\left(P^{+}\right)-\rho_{\alpha \varepsilon}\left(P^{+}\right)$and $\rho_{\alpha \alpha}\left(Q^{-}\right)-\rho_{B B}\left(Q^{-}\right)$ (at the appropriate magnetic fields), which are defined as the polarizations on $\mathrm{P}^{+}\left(<\mathrm{S}_{1 \mathrm{q}}\right\rangle$ ) and 


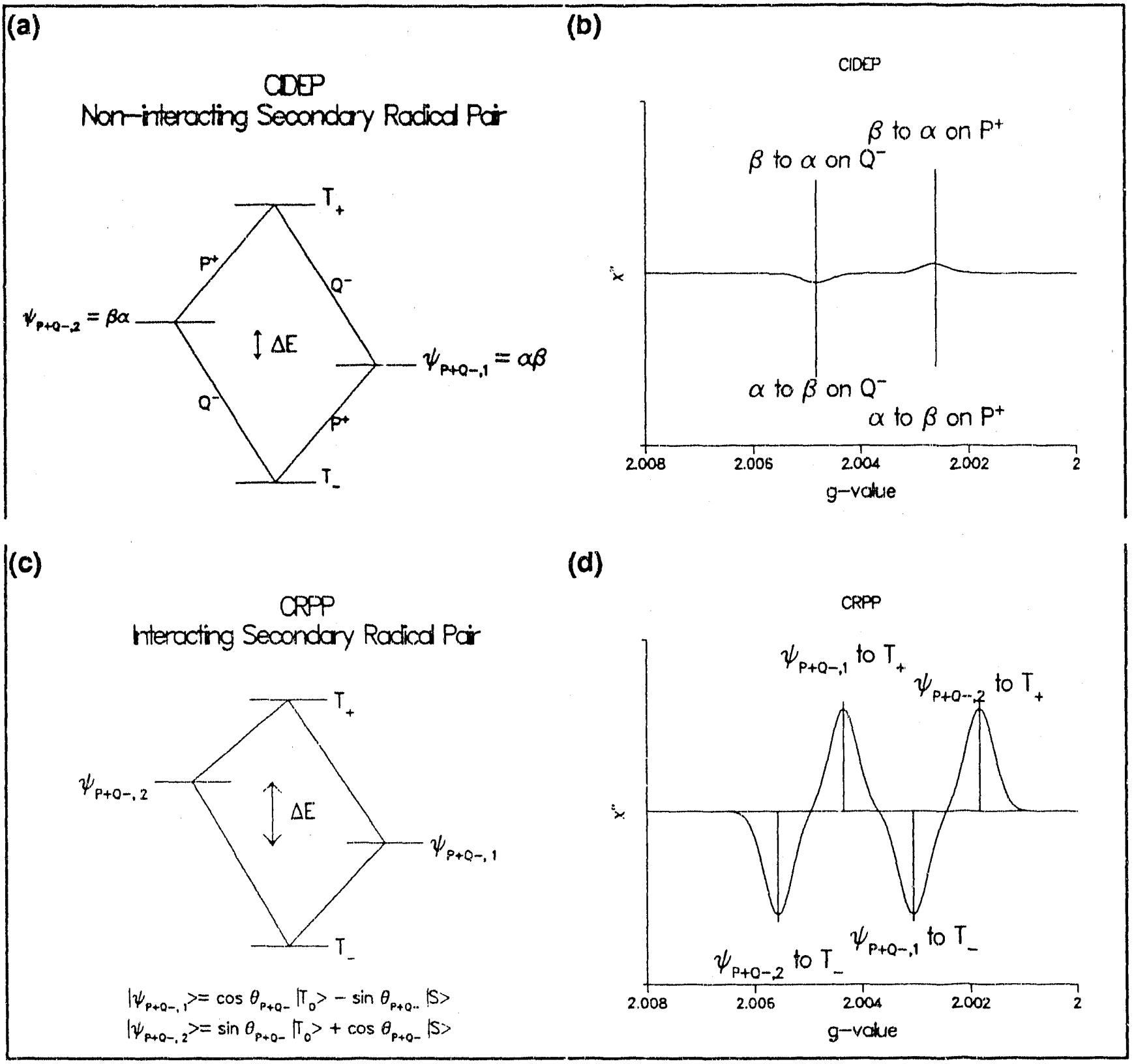

Figure 2(a) The energy levels of the $\mathrm{P}^{+} \mathrm{Q}^{-}$radical pair in the absence of interactions. $\Delta E=$ $\left(g_{p^{+}, \text {ef }}-g_{Q^{-, e f}}\right) B B_{0}+\Sigma_{i} a_{i}^{p^{+}} m_{i}^{p^{+}}-\Sigma_{k} a_{k}^{Q^{-}} m_{\mathrm{k}}^{f^{-}}$. $2(b)$. An illustration of the effects of interactions on $\mathrm{P}^{+} \mathrm{I}^{-}$in the absence of interactions on $\mathrm{P}^{+} \mathrm{Q}^{-}$. Dpi $=-4$ Gauss, $\mathrm{Jpi}=7$ Gauss, $\mathrm{g}_{\mathrm{P}^{+}}=2.0026, \mathrm{~g}_{\mathrm{r}}$ $=2.0035, g_{Q^{-}}=2.0048$. For simplicity no g-anisotropy has been included in the simulations shown; $g_{i, e f}=g$ for pigment $i$ at all angles. In the figure, the sticks represent the calculated position and intensity of the transitions for a particular set of proton spins. The broadened lines represent the Gaussian distribution expected from an ensemble of a large number of proton spins. 2(c) The eigenstates of the $\mathrm{P}^{+} \mathrm{Q}^{-}$radical pair with or without interactions on $\mathrm{P}^{+} \mathrm{I}^{-}$. $\Delta E=\left[\left(2 \mathrm{~J}_{\mathrm{PQ}}^{2}-D_{\mathrm{PQ}}^{2}\left(\cos B_{\mathrm{P}+\mathrm{Q}}-1 / 3\right)\right)^{2}+(\Delta E \text { from } 2(\mathrm{a}) \text { above })^{2}\right]^{1 / 2}$. 2(d) A simulated spectrum of an interacting $\mathrm{P}^{+} \mathrm{Q}^{-}$radical pair. The example is for no interactions on $\mathrm{P}^{+} \mathrm{I}^{-}$and for identical effective $\mathrm{P}^{+}$and $\mathrm{I}^{-} \mathrm{g}$ values.

$\left.\mathrm{Q}^{-}\left(<\mathrm{S}_{2 \mathrm{z}}\right\rangle\right)$ respectively. The polarization on $\mathrm{P}^{+}$can be shown to be equal, for an initial singlet state, to the familiar $\rho_{S T_{0}}+\rho_{T_{0} s}$ defined as $\rho_{x}$ in the vector model treatment. ${ }^{11,12}$ Because the polarization develops on the primary radical pair and is transferred, the total polarization on $\mathrm{P}^{+} \mathrm{Q}^{-}$is the integral over time of the polarization weighted by the probability of the transfer 
occurring at a particular time (effectively with a particular polarization), $1 / \tau \cdot \theta^{-t / \tau}$, where $\tau$ is the inverse of the electron transfer rate from $\mathrm{P}^{+} \rho^{-}$to $\mathrm{P}^{+} \mathrm{Q}^{-}$.

Interacting radical pair spectra, however, are not proportional to the polarization -$\rho_{k e}\left(P^{+}\right)-\rho_{B \theta}\left(P^{+}\right)$at the magnetic field strength for enhanced absorption and emission on $\mathrm{P}^{+}$, and $\rho_{s e}\left(Q^{-}\right) \rho_{B \theta}\left(Q^{-}\right)$at the magnetic field strength for enhanced absorption and emission on $Q^{-}$. Even if we consider a parallel definition of the polarization in which it is equal to the difference in population of the eigenstates of the Hamiltonian, the spectrum is not directly proportional. Since the transitions from $\Psi_{1}$ to $T_{+}$(the spin on either $\mathrm{P}^{+}$or $\mathrm{Q}^{-}$goes from $B$ to $\alpha$ ) and from $\Psi_{2}$ to $T_{\text {. }}$ are at different energies, the transitions occur at different magnetic fields (see figure 2(d)). The transitions are now weighted by unequal transition probabilities, as well. This balances the population differences for the CRPP only case. In the absence of interactions on the primary radical pair, the $\mathrm{P}^{+}$transition lines will be split, but they will have equal intensities. Even without interactions on the primary radical pair, however, the line intensities will not cancel, since all four allowed transitions now occur at different magnetic field strengths. The spectrum is not directly proportional to the polarization as it is for noninteracting secondary radical pairs. See figure $2(c, d)$.

When interactions on both $\mathrm{P}^{+}{ }^{-}$and $\mathrm{P}^{+} \mathrm{Q}^{-}$are treated, the population differences are only partially balanced by the unequal transition probabilities. The effects of the interactions between $P^{+}$and $Q^{-}$on the populations of the secondary radical pair's eigenstates are uffset by the transition probabilities. The popu'ation differences due to the interactions between $P^{+}$ and ${ }$ are not compensated by the transition probabilities. Thus, the polarization transferred from $\mathrm{P}^{+} I^{-}$will be reflected as unequal $\mathrm{P}^{+} \mathrm{Q}^{-}$transition intensities. See figure $3(b)$--the adshed speürum.

It should be understood that we are not considering here a case in which some $\mathrm{P}^{+} \mathrm{Q}^{-}$radical pairs interact while others do not. In order to include this case we would calculate both the non-interacting radical pair spectrum (CIDEP) and the interacting radical pair spectrum (CRPP). We could then assign a fraction of the ensemble as interacting, with all others non-interacting, and sum the weighted spectra. ${ }^{13}$ (See figure 3.) We think a distribution of pairs would be unlikely in the bacterial reaction center's highly structured protein environment. In this paper, we are concerned with the sequence of interacting radical pairs $\mathrm{P}^{+} \mathrm{I}^{-}-\mathrm{P}^{+} \mathrm{Q}^{-}$. The difference between a spectrum resulting from a distribution of radical pairs and a spectrum resulting from a sequence of radical pairs is illustrated in fig $3(b)$.

We are concerned with the similarities between the CIDEP and CRPP calculations because we use the CRPP treatment to calculate the spectrum even in the case of non-interacting radical pairs. We do not calculate $\rho_{S T_{0}}+\rho_{T_{0} S}$ as is generally done for non-interacting radicals; 

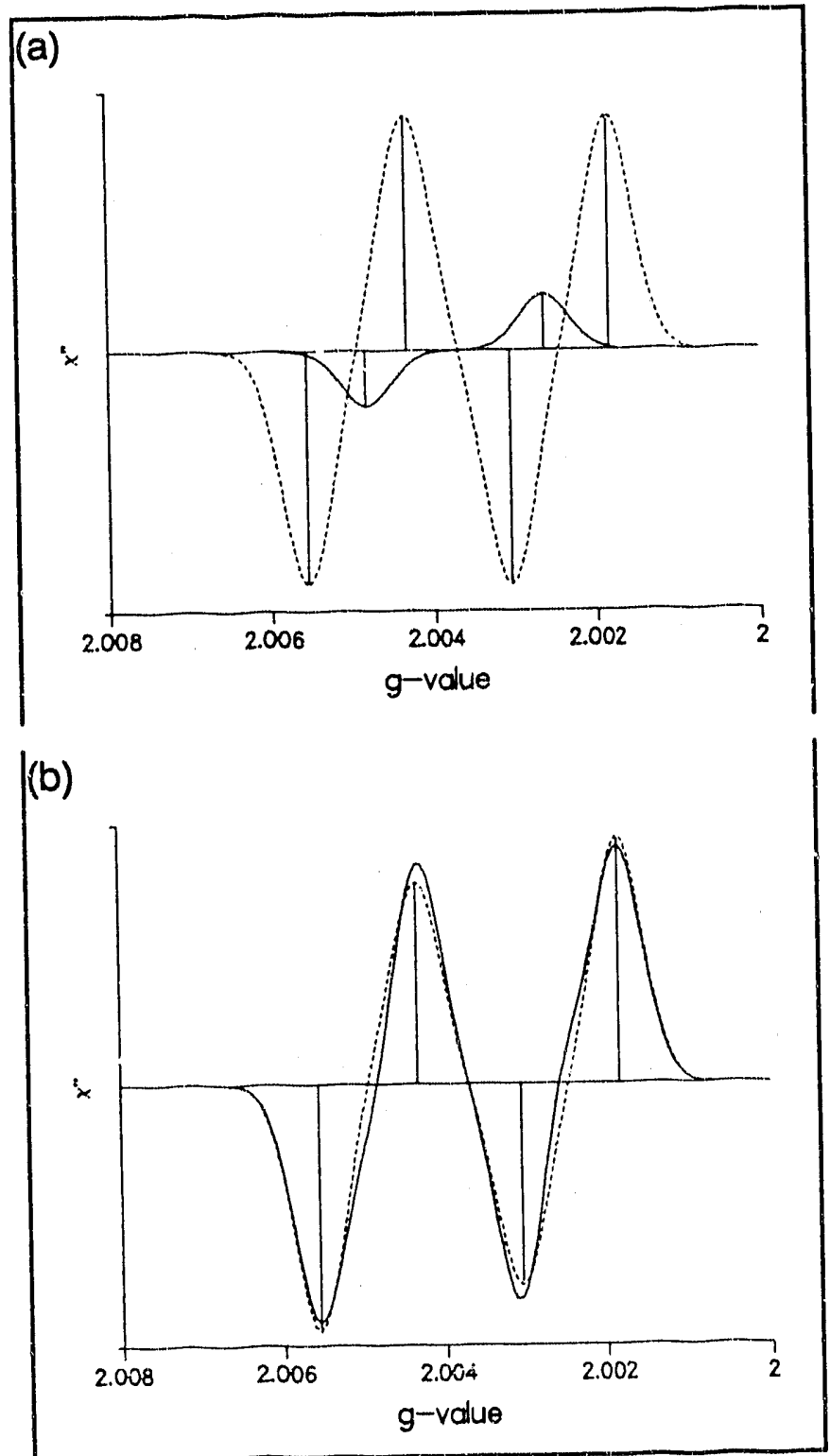

Figure 3 (a) shows an overlay of the CIDEP and the CRPP spectra. The solid line is the CIDEP spectrum, and the dashed line is the CRPP spectrum. $3(\mathrm{~b})$ shows the difference between an ensemble of radical pairs ( $50 \%$ interacting and $50 \%$ non-interacting) and a sequence of radical pairs. The solid line is the ensemble arid the dashed line is the sequence.

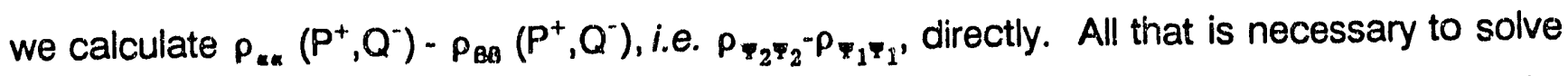
the problem formally, then, is to find the eigenstates of the Hamiltonian and to solve the density matrix equations for the two radical pairs.

Since we wish to simulate the effect of both the polarization developed on the first radical pair and the effect of the splitting due to the exchange and dipolar interactions on the second radical pair, we must include the electron transfer in the density matrix equations. We elect to treat the two radical pairs as separate systems and solve two coupled first order difierential equations, (2) and (3), for a two-by-two density matrix rather than one equation for a four-byfour density matrix. 


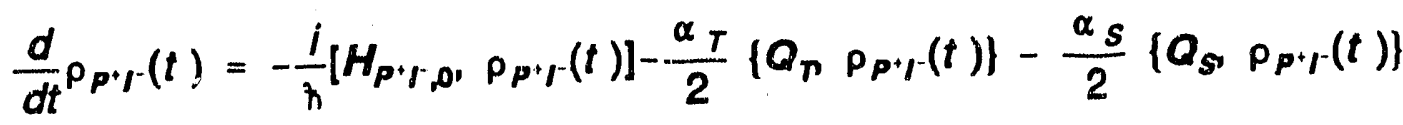

$$
\begin{aligned}
& \frac{d}{d t} \rho_{P+Q}(t)=-\frac{i}{\hbar}\left[H_{P+Q}, \rho_{P^{+} Q^{-}}(t)\right]+k_{Q} \rho_{P+r}(t)
\end{aligned}
$$

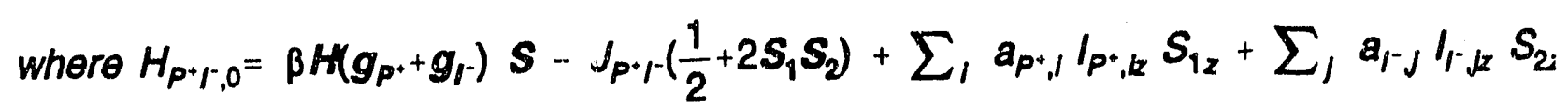

$$
\begin{gathered}
+\frac{1}{2}\left(3 D_{P^{+}+}\left(\cos ^{2} \beta_{P^{+} Q^{-},+}-\frac{1}{3}\right)\left(s_{z}^{2}-\frac{1}{3} s^{2}\right)\right), \\
\alpha_{T}=k_{T^{+}} k_{Q} \text { and } \alpha_{s}=k_{s^{+}} k_{Q}
\end{gathered}
$$

The addition of the kinetic terms to the density matrix equations are due to first order electron transfers to the ground state (from singlet radical pair populations only with rate $k_{S}$ ), the lowest lying molecular triplet (from triplet radical pair populations only with rate $\mathrm{k}_{\mathrm{T}}$ ), and to $\mathrm{P}^{+} \mathrm{Q}^{-}$(from both singlet and triplet radical pairs with rate $k_{Q}$ ). The curled braces indicate the anticommutator, and $Q_{S}$ and $Q_{T}$ are the projection operators for the singlet and triplet states respectively. As is usual, $B$ above is the Boinr magneton; $a_{p^{+}, \mathrm{i}}$ are the hyperfine coupling constants on $\mathrm{P}^{+}$. These hyperfine iriteractions are modeled with a Gaussian. Individual combinations of hyperfine interactions are represented as evenly spaced points along a Gaussian lineshape. $\beta_{P^{+}+, H}$ is the angle between the dipolar vector and the magnetic field vector, $\mathbf{H}$, and the various $\mathbf{I}^{\prime}$ s and $\mathbf{S}$ 's are the usual spin operators. $D_{\mathrm{P}^{+} \mathrm{I}}$-represents the strength of the dipolar interaction, and $J_{\mathrm{p}+\mathrm{I}}$ is the strength of the exchange interaction. The dipolar interaction is assumed to be axially symmetric, and the exchange interaction is considered to be isotropic. $H_{P \cdot Q^{-0, Q}}\left(=H_{P^{+} Q^{-}}\right)$is defined in a fashion strictly analogous to the definition of $H_{P+r, Q}$ in (4).

In order to solve (2) and (3) we define a quantity

$$
H_{P+Y}=H_{P+r, 0}-\frac{i \hbar}{2}\left(\alpha_{T} Q_{T}+\alpha_{s} Q_{S}\right)
$$

Then,

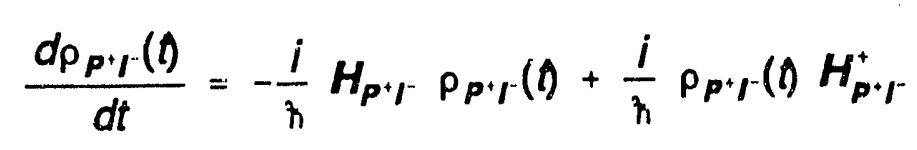

where $\boldsymbol{H}_{\boldsymbol{P}^{+},}^{+}$is the transpose of the Hamiltonian. The solution of this equation is easily demonstrated to $b^{14}$

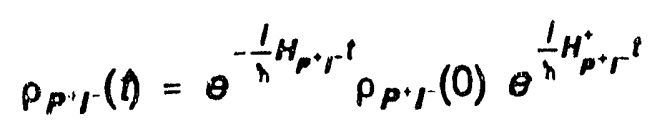


This expression for $\rho_{p+1}$ is then substituted into the solution for (3).

Equations (2) and (3) represent two coupled, first order, linear, non-homogenous differential equations of the form $d x / d t+a(x) x=f(x)$. For radical pairs in liquid solution $x$ and $f(x)$ are functions of distance as well as time and the total derivative is far more complex. In addition, $f(x)$ may be of a non-integrable form or $a(x)$ may be a non-constant expression. For these reasons, the equivalent of (3) in non-photosynthetic systems generally does not have an analytic solution. For photosynthetic systems, however, the protein matrix determines the distance and orientation of $P, I$, and $Q$, as well as the other pigments. $J$ and $D$ are discrete functions of distance with only two values defined. One of these is represented by the subscript $\mathrm{P}^{+} \mathrm{I}^{-}$, and the other by the subscript $\mathrm{P}^{+} \mathrm{Q}^{-}$. An analytical expression for (3) exists as demonstrated below.

Consider:

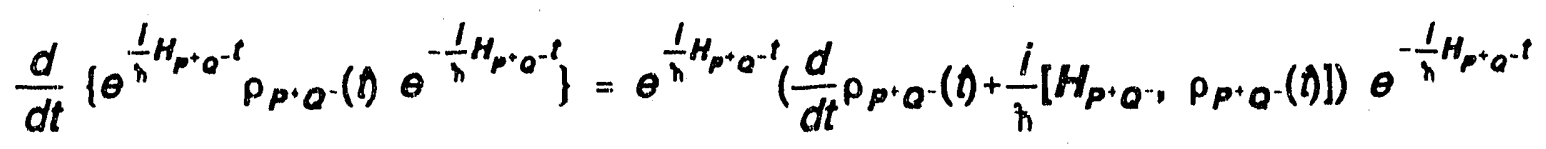

Then, from (3) above

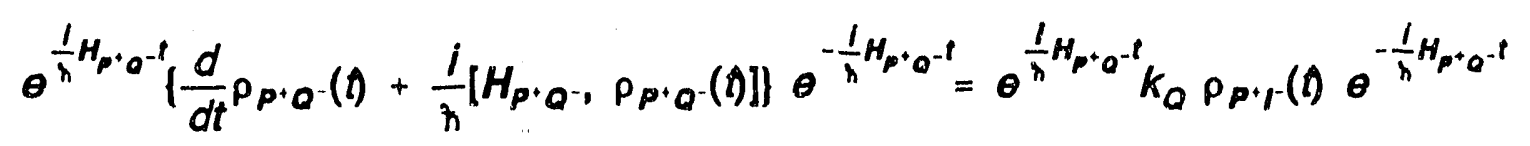

Equating the left hand side of (5) with the right hand side of (6), integrating from 0 to $t$, and applying the appropriate rotation yields

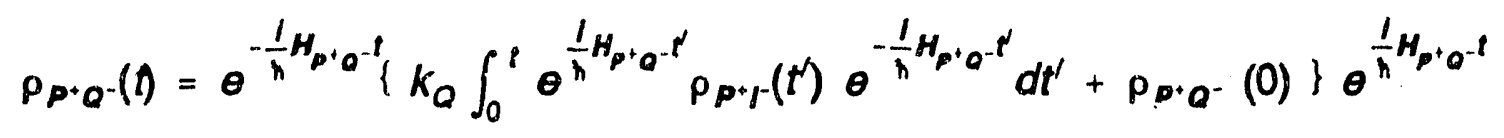

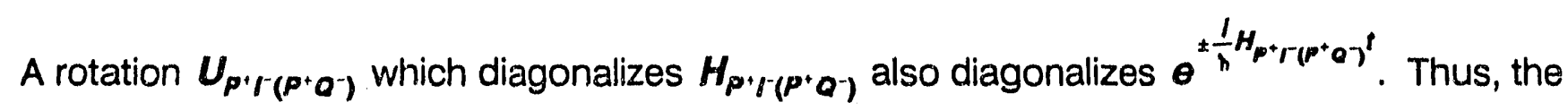

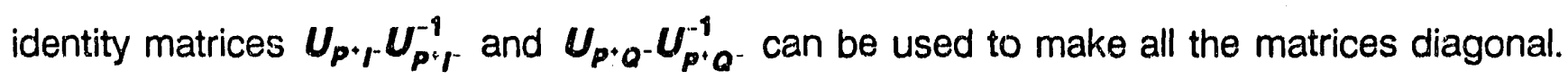
Diagonalizing all matrices and substituting in the diagonalized form of $\rho_{p^{+}+}(t)$ derived above gives

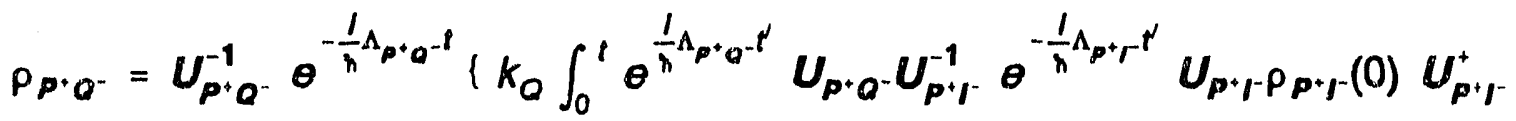

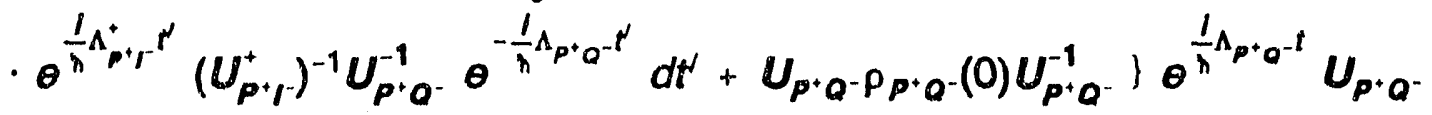




$$
\begin{aligned}
& U_{1}=\left(\begin{array}{cc}
\cos \theta_{1} & -\sin \theta_{1} \\
\sin \theta_{1} & \cos \theta_{1}
\end{array}\right) \quad \text { and } \quad \Lambda_{1}=\left(\begin{array}{cc}
\lambda_{41} & 0 \\
0 & \lambda_{12,}
\end{array}\right) \quad i=P^{+} I^{-}, P^{+} Q^{-} \\
& \lambda_{h 1}=E_{\psi_{L 1}}=\gamma_{1}-\frac{1}{2} \omega_{1} \quad \cos \theta_{1}=\frac{2 Q}{\sqrt{2 \omega\left(\Delta E_{L S T_{0}}+\omega\right)}} \\
& \lambda_{l, 2}=E_{\downarrow_{12}}=\gamma_{1}+\frac{1}{2} \omega_{1} \\
& \sin \theta_{1}=\sqrt{\frac{\Delta E_{l S T_{0}}+\omega_{1}}{2 \omega_{1}}} \\
& E_{P^{+} I^{\prime}, T_{+}}=\frac{1}{2}\left(g_{P^{+}}+g_{l \cdot}\right)+\frac{1}{2}\left(\sum_{l} a_{l}^{P^{+}} m_{l}^{P^{+}}+\sum_{l} a_{j}^{l^{\prime}} m_{l}^{l^{\prime}}\right)+\frac{D_{P^{+} l^{-}}}{2}\left(\cos ^{2} \beta_{P^{+} I^{\prime}, B_{0}}-\frac{1}{3}\right)-J_{P^{+} I^{-}} \\
& E_{P^{+} l^{\prime}, T_{-}}=-\frac{1}{2}\left(g_{P^{+}}+g_{l^{-}}\right)-\frac{1}{2}\left(\sum_{l} a_{l}^{P^{+*}} m_{l}^{P^{+}}+\sum_{l} a_{l}^{l^{-}} m_{l}^{l^{-}}\right)+\frac{D_{P^{+} l^{-}}}{2}\left(\cos ^{2} \beta_{P^{+} l^{\prime}, B_{0}}-\frac{1}{3}\right)-J_{P^{+} l^{-}} \\
& \omega_{i}=\sqrt{\Delta E_{h}^{2} S T_{0}+\left(2 Q_{i}\right)^{2}} \\
& \gamma_{P^{+1}+}=-\frac{1}{2}\left[\frac{i}{2}\left(\alpha T^{+\alpha} \alpha_{S}\right)+D_{P^{+1}-}\left(\cos ^{2} \beta_{P^{+} /, B_{0}}-\frac{1}{3}\right)\right] \\
& \gamma_{P^{*} Q^{-}}=-\frac{1}{2} D_{P^{+} Q^{-}}\left(\cos ^{2} \beta_{P^{+} Q^{-}, B_{0}}-\frac{1}{3}\right)
\end{aligned}
$$

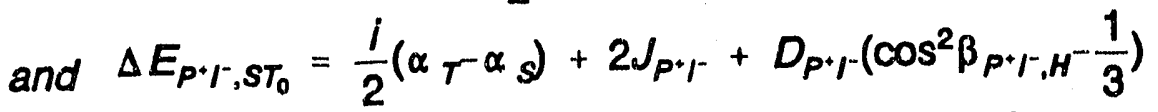

$$
\begin{aligned}
& \Delta E_{P^{+} O^{-}, S T_{0}}=2 J_{P^{*} Q^{-}}+D_{P^{*} Q^{-}}\left(\cos ^{2} \beta_{P^{+} Q^{-}, H^{-}}-\frac{1}{3}\right) \\
& Q_{P^{*} l^{-}}=\frac{1}{2}\left(g_{P^{*}}-g_{l}\right) \beta B_{0}+\frac{1}{2}\left(\sum_{l} a_{l}^{p^{*}} m_{l}^{p^{*}}-\sum_{l} a_{l}^{l^{\prime}} m_{l}^{l^{\prime}}\right) \\
& Q_{P^{*} Q^{-}}=\frac{1}{2}\left(g_{P^{*}}-g_{Q^{-}}\right) \beta B_{0}+\frac{1}{2}\left(\sum_{l} a_{l}^{P^{+}} m_{l}^{P^{*}}-\sum_{k} a_{k}^{Q^{-}} m_{k}^{Q^{-}}\right)
\end{aligned}
$$

$B_{0}$ is the strength of the magnetic field vector, $H$, which was taken along the $z$ axis above. The energies for the triplet states on $\mathrm{P}^{+} \mathrm{Q}^{-}$are identical to those for $\mathrm{P}^{+} I^{-}$with all occurences of $\mathrm{I}^{-}$ replaced with $Q^{-}$.

Our interest, however is in the density matrices in the eigenspaces of the Hamiltonians. Therefore we apply the rotation which diagonalizes the Hamiltonian to each density matrix. In the following we show the rotation $\boldsymbol{U}_{\boldsymbol{P}^{+} \boldsymbol{Q}^{-}}$applied to the density matrix on $\mathrm{P}^{+} \mathrm{Q}^{-}$.

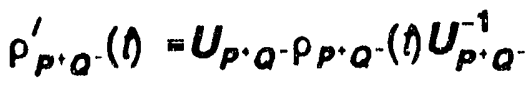

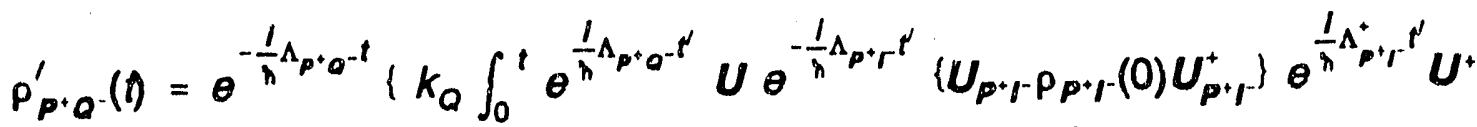

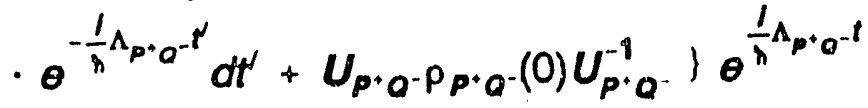




$$
\text { where } U=U_{P^{+} Q^{-}} U_{p^{+}+I^{-1}}^{-1} \text { and } U^{+}=\left(U_{\left.p^{+}+\right)^{+}}^{-1} U_{p^{+} Q^{-}}^{-1}\right.
$$

Working out all the integrations and matrix multiplications gives for no density in the secondary radical pair state on initial absorption of light $\left(\rho_{\mathbf{P}_{\mathbf{P}+Q-2}}(0)=[0]\right)$, and a pure singlet radical pair precursor

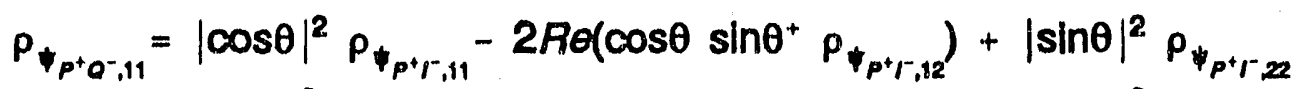

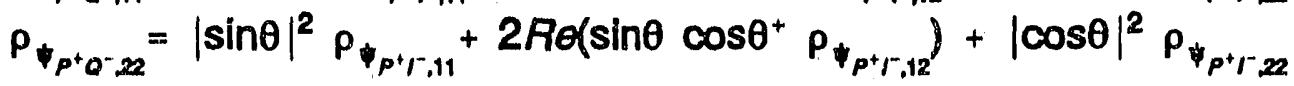

for the diagonal elements of the density matrix, i.e., the populations of the eigenstates which are mixtures of $S$ and $T_{0}$.

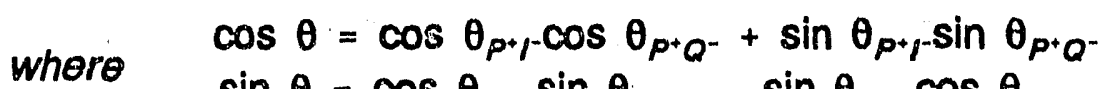

$$
\begin{aligned}
& \sin \theta=\cos \theta_{P^{+}-}-\sin \theta_{P^{+} Q^{-}}-\sin \theta_{P^{+}+} \cos \theta_{P^{+} Q^{-}}
\end{aligned}
$$

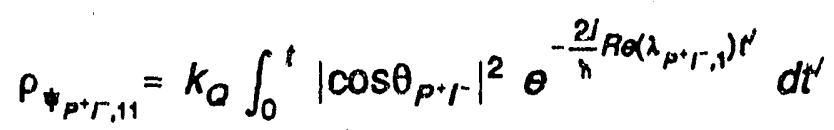

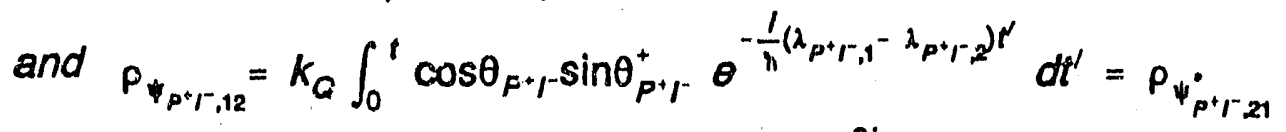

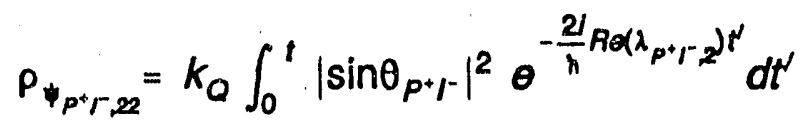

The $\rho_{\psi_{p+r}, 11}$ are the elements of the density matrix for the primary radical pair in its eigenspace after they have been transferred to the secondary radical pair.

As described earlier the intensity of each transition is calculated from the product of the difference in population and the transition probability. The $T_{+, P^{*} Q^{-}}$and $T_{-, P^{*} Q^{-}}$eigenstates are unpopulated; the energy difference between these states and the singlet state is too high for mixing. The populations of the $\Psi_{P^{+} O^{-1}, 1}$ and $\Psi_{P^{+} Q^{-, 2}}$ are listed above (eq. 11). The transition probability between eigenstates $a$ and $b$ is given by $\left\langle b\left|S_{x}\right| a\right\rangle$. Only the four transitions from $\Psi_{P^{+} Q^{-, 1}}$ to $T_{ \pm, P^{+} Q^{-}}$and from $\Psi_{P^{+} Q^{-, 2}}$ to $T_{ \pm, P^{+} Q^{-}}$are allowed. The transition probabilities for transitions from the $\psi_{P+Q, 1}$ eigenstate are proportional to $\left|\cos \theta_{P^{+} Q^{-}}\right|^{2}$ and those for transitions from the $\psi_{P^{+} Q^{-}, 2}$ are proportional to $\left|\sin \theta_{P^{+} Q^{-}}\right|^{2}$.

The progression from a CIDEP spectrum to a CRPP spectrum is illustrated in figure 4. Although good estimates of all parameters: the magnetic interactions on both radical pairs, the $\mathbf{g}$ tensor on $\mathrm{P}^{+}, \mathrm{r}^{-}$, and $\mathrm{Q}^{-}$, the linewidths for $\mathrm{P}^{+}, \mathrm{I}^{-}$and $\mathrm{Q}^{-}$, the orientation of the pigments 


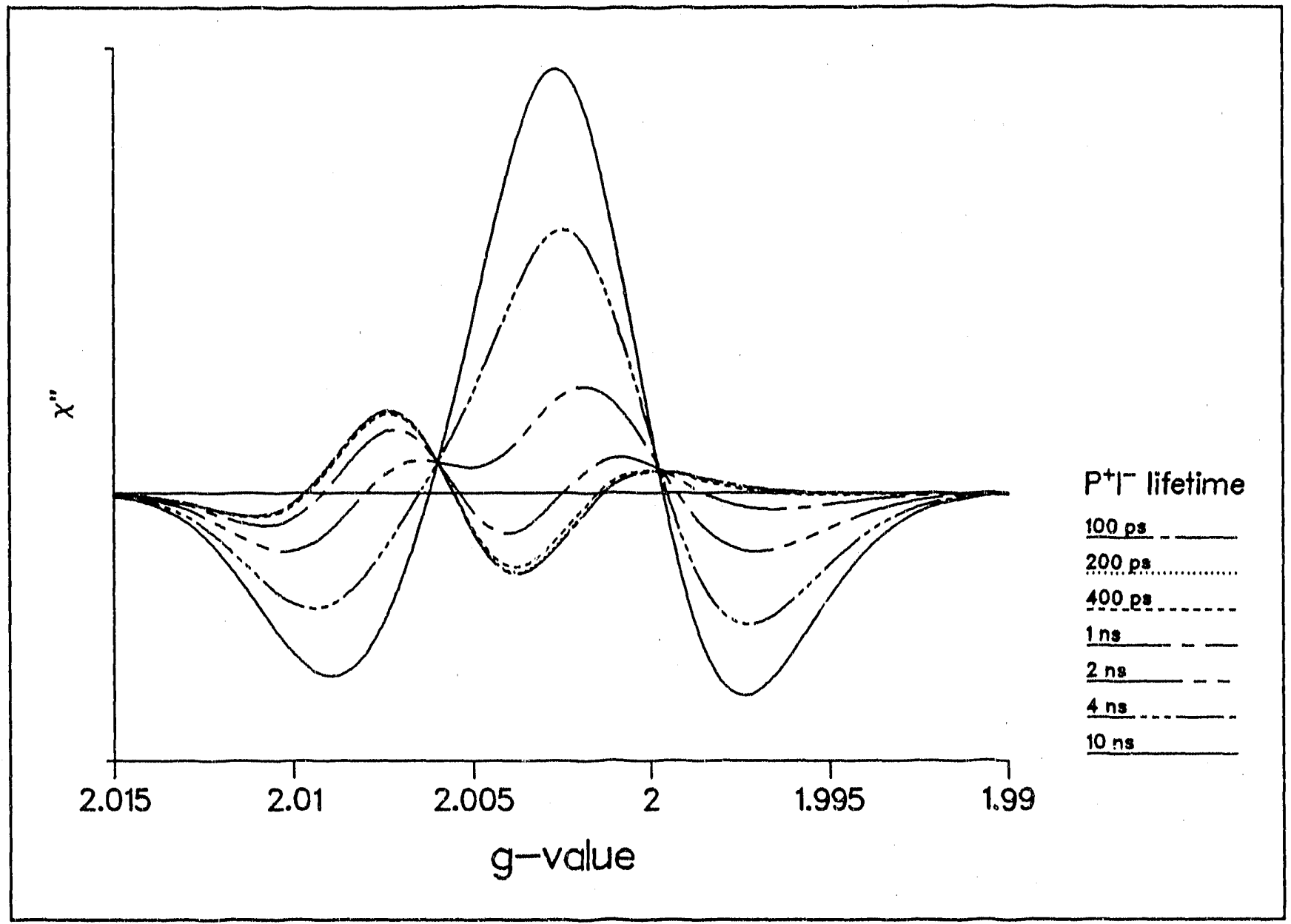

Figure 4

The radical pair spectrum as a function of the lifetime of the primary radical pair.

Reasonable sets of parameters were used to calculate the spectra in figure 4. \# angles: 21X21,

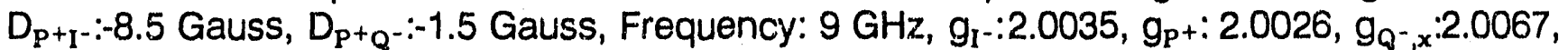
$g_{Q^{-}, y}: 2.0056, g_{Q^{-}, z^{2}}: 2.0024, J_{P^{+} I^{-}}: 7$ Gauss, $J_{P^{+} Q^{-}}: 0$ Gauss, $k_{Q}: 1052.51 .5 .25$ and $.1 \mathrm{GHz}^{*} \mathrm{k}_{\mathrm{S}}$ : $0 \mathrm{GHz}, \mathrm{k}_{\mathrm{T}}: 0 \mathrm{GHz}$, \# hyperfine combinations $\mathrm{I}^{-}: 21 ; \mathrm{P}^{+}: 21 ; \mathrm{Q}^{-}: 21$, time: $100 \mu \mathrm{s}$ after creation of $\mathrm{P}^{+} \mathrm{I}^{-}\left(\mathrm{T}_{1}\right.$ processes have been neglected), $\mathrm{I}^{-}$linewidth: 13.5 Gauss, $\mathrm{P}^{+}$linewidth: 9.5 Gauss, $Q^{-}$linewidth: 6 Gauss, Angle between $D_{p+1}$ and $Q_{z}$ axis: $10.7^{\circ} ; Q_{x}$ axis: $2.68^{\circ}$, Angle between

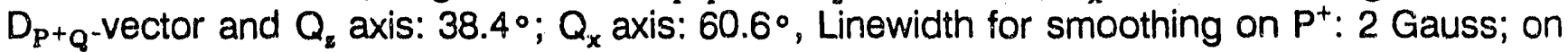
$Q^{-}: 2$ Gauss.)

( $P, I$ and $Q)$ within the reaction center, and the kinetic rate constants are available for reaction centers, any set of these parameters could be varied within a small range in order to obtain a good fit to all the experimental data. Specifically, we emphasize here the effect on the EPR spectrum of changing the rransfer rate from the primary radical pair to the secondary radical pair, $k_{\mathrm{Q}}$. In a previous paper we gave a limited description of this effect in addition to a description of the effect of changes in the dipolar term on the second radical pair and a description of the effect of changes in the transfer rate from the primary radical pair to the molecular triplet on $\mathrm{P}, \mathrm{k}_{\mathrm{T}}{ }^{15}$

If the rate constant $k_{Q}$ is large, $(200 \mathrm{ps})^{-1}$, the transfer from $\mathrm{P}^{+} \mathrm{I}^{-}$to $\mathrm{P}^{+} \mathrm{Q}^{-}$is fast, a spectrum resembling the CRPP spectrum is expected. The density matrix on the primary radical pair 
does not have sufficient time to develop triplet character so any observed polarization is due to the CRPP mechianism. If the rate constant $k_{Q}$ is small, $(10 \mathrm{~ns})^{-1}$, the transfer from $\mathrm{P}^{+} \mathrm{I}^{-}$to $P^{+} Q^{-}$is slow, we expect to sae a spectrum which reflects both the transferred polarization and the splitting due to the megnetic interactions on $\mathrm{P}^{+} \mathrm{Q}^{-}$. When the magnetic interactions on $\mathrm{P}^{+} \mathrm{Q}^{-}$ are smail, the EPR lines, which are broadened by g-anisotropy and hyperfine interactions, effectively cancel, and the spectrum resembles the CIDEP spectrum. When the EPR lines are broad it is possible for the CIDEP spectrum to have a greater intensity than the CRPP spectrum. Figure 4 shows calculated spectra as a function of $k_{Q}$. Intermediate cases can only be treated by including interactions on both radical pairs.

\section{Acknowledgements}

We thank Jau Tang, David Budi!, Peter Gast and Laura Feezel for many helpful discussions.

This work was supported by the U.S. Department of Energy, Office of Basic Energy Sciences, Division of Chemical Sciences under contract W-31-109-Eng-38. 


\section{References}

1. C. Kirmaier, D. Holton: Photosyn. Res, 13,225 (1987)

2. C. Kirmaier, D. Holton, W.W. Parson: Blochim. Biophys. Acta 810, 33 (1985)

3. P. Gast: Thesis, University of Leiden, Leiden, The Netherlands (1982)

4. A. degroot, P. Gast, A.J. Hoff: AdV. in Photosyn. Res. Vol. I, C. Sybesma, ed. (Martinus Nijhoff/Dr. W. Junk, The Hague 1984) Pp. 215-218

5. P.J. Hore, E.T. Watson, J. Boiden Pederson, F..J. Hoff: Biochim. Biophys. Acta 849,70 (1986)

6. P.J. Hore, D.A. Hunter, C.D. MaKie, A.J. Hoff: Chem. Phys. Lett. 137, \$95 (1987)

7. J.D. MCElroy, G. Feher, D.C. Mauzerall: Biochim. Biophys. Acta 267, 363 (1972)

8. C. Kirmaier, D. Holten, D.J. Debus, G. Feher, M.Y. Okamura: Proc. Nat1. Acad. SCi. USA 83,6407 (1986)

9. M.R. Gunner, D.E. Robertson, R.L. LoBrutto, A.I. McLaughlin, P.L. Dutton: Progress in Photosynthesis Rusearch Vol. 1, J. Biggins, ed. (Martinus Nijhoff, Dordrecht, 1987) pp. 217-220

10. L.I. Feezel, P. Gast, U.H. Smith, M.C. Thurnauer: Biochim. Biophys. Acta 974, 149 (1989)

11. J.R. Norris, A.L. Morris, M.C. Thurnauer, J. Tang: J. Chem. Phys. 92,4239 (1990)

12. I. Monchick and F.J. Adrian, J. Chem. Phys. 68, 4376 (1978)

13. G.L. ClosB, M.D.E. Forbes, J.R. Norris: J. Phys. Chem. 91, 3592 (1987)

14. J. Tang, J.R. Norris: unpublished results.

15. M.C. Thurnauer, L.L. Feezel, A.I. Morrib, U. Smith, J.R. Norris: Current Research in Photosynthesis Vol. 1, M. Baltscheffiky, ed. (Kluver Academic Publishers, Dordrecht, 1989) pp. 181-184 


